\title{
Erratum: Validation of 1-hour post-thyroidectomy parathyroid hormone level in predicting hypocalcemia
}

Trung N Le ${ }^{1 *}$, Paul D Kerr ${ }^{2}$, Donna E Sutherland ${ }^{2}$ and Pascal Lambert ${ }^{3}$

\section{Erratum}

Following publication of our article [1], it has been brought to our attention that we have incorrectly stated the PTH levels for the low risk and high risk patients in the Methods section in the Abstract.

The correct sentence should be:

Based on our previous work, patients were stratified into either a low risk group (PTH $\geq 12 \mathrm{pg} / \mathrm{ml})$ or a high risk group $(\mathrm{PTH}<12 \mathrm{pg} / \mathrm{ml})$.

We would like to apologise for any confusion caused to our readers.

\section{Author details \\ 'Otolaryngology Head \& Neck Surgery, University of Manitoba, Health Sciences Centre, GB421-820 Sherbrook Street, Winnipeg, MB R3A 1R9, Canada. ${ }^{2}$ Head and Neck Oncology, University of Manitoba, Health Sciences Centre, GB421-820 Sherbrook Street, Winnipeg, MB R3A 1R9, Canada. ${ }^{3}$ Health Outcomes Analyst, Cancer Care Manitoba, ON2114-675 McDermot, Avenue, Winnipeg, MB R3E 0V9, Canada.}

Received: 2 May 2014 Accepted: 14 October 2014

Published online: 20 November 2014

\section{Reference}

1. Le TN, Kerr PD, Sutherland DE, Lambert P: Validation of 1-hour post-thyroidectomy parathyroid hormone level in predicting hypocalcemia. J Otolanyngol Head Neck Surg 2014, 43:5.

\section{doi:10.1186/s40463-014-0042-6}

Cite this article as: Le et al: Erratum: Validation of 1-hour post-thyroidectomy parathyroid hormone level in predicting hypocalcemia. Journal of Otolaryngology - Head and Neck Surgery 2014 43:42.

\footnotetext{
* Correspondence: trungngocle@gmail.com

${ }^{1}$ Otolaryngology Head \& Neck Surgery, University of Manitoba, Health Sciences Centre, GB421-820 Sherbrook Street, Winnipeg, MB R3A 1R9 Canada

Submit your next manuscript to BioMed Central and take full advantage of:

- Convenient online submission

- Thorough peer review

- No space constraints or color figure charges

- Immediate publication on acceptance

- Inclusion in PubMed, CAS, Scopus and Google Scholar

- Research which is freely available for redistribution 